\title{
Photon-Photon and Photon-Hadron Physics at Relativistic Heavy Ion Colliders
}

\author{
K. Hencken ${ }^{\mathrm{a}}$, P. Stagnoli ${ }^{\mathrm{a}}$, D. Trautmann ${ }^{\mathrm{a}}$ and G. Baur ${ }^{\mathrm{b}}$ \\ a Universität Basel, Klingelbergstr. 82, CH-4056 Basel \\ ${ }^{\mathrm{b}}$ Forschungszentrum Jülich,D-52425 Jülich
}

\section{Introduction}

The parton model is very useful to study scattering processes at very high energies. For example, nuclei consist of nucleons, which in turn consist of quarks and gluons, photons consist of lepton pairs, electrons of photons, etc.. Relativistic nuclei have photons as an important constituent at low enough virtuality $Q^{2}=$ $-q^{2}$ of the photon, due to the coherent action of all charges in the nucleus. The coherence condition limits the virtuality of the photons to

$Q^{2} \lesssim 1 / R^{2}$

where the radius of a nucleus is given approximately by $R=1.2 \mathrm{fm} A^{1 / 3}$, with $A$ the nucleon number. From the kinematics of the process one has $Q^{2}=\frac{\omega^{2}}{\gamma^{2}}+q_{\perp}^{2}$. This limits the maximum energy of the quasireal photon and the perpendicular component of its momentum to

$\omega<\omega_{\max } \approx \gamma / R, \quad q_{\perp} \lesssim 1 / R$,

where $\gamma$ is the Lorentz factor of the projectile. The ratio $x=\omega / E$, where $E$ denotes the energy of the nucleus, is smaller than $x<x_{\max }=1 /\left(R M_{N} A\right)$ (we always use $\hbar=c=1)$. We find $x \lesssim 10^{-3}$ for Ca ions and $\lesssim 10^{-4}$ for $\mathrm{Pb}$ ions.

The collisions of $e^{+}$and $e^{-}$has been the traditional way to study $\gamma \gamma$-collisions. Similarly photon-photon collisions can also be observed in hadron-hadron collisions. Of course, the strong interaction of the two nuclei has to be taken into consideration. Up to now hadron-hadron collisions have not been used for two-photon physics. An exception can be found in [1], where the production of $\mu^{+} \mu^{-}$pairs at the ISR was studied. The special class of events was selected, where no hadrons are seen associated with the muon pair. In this way one makes sure that the hadrons do not interact strongly with each other, i.e., one is dealing with peripheral collisions (impact parameters $b>2 R$ ).

Due to the coherent action of all protons the photon flux in heavy ion collisions is large $\left(\sim Z^{2}\right)$ and therefore of interest for photon-photon and photon-hadron physics. This subject has been studied for several years. Recent reviews of this topic are [2, 3]. The "Relativistic Heavy Ion 


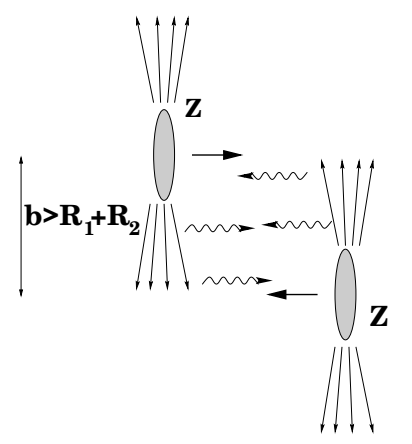

Figure 1. Two fast moving electrically charged nuclei are an abundant source of (quasireal) photons. They can collide with each other and with the other nucleus. For peripheral collisions with impact parameters $b>2 R$, this is useful for photonphoton and photon-nucleus collisions.

Collider" (RHIC) will have a program to investigate such collisions experimentally [1] and similar programs are discussed at LHC 58 81. At RHIC $(\gamma \approx 100)$ the equivalent photon spectrum extends up to several GeV. Therefore the available invariant mass range is up to about the mass of the $\eta_{c}$. When the "Large Hadron Collider" will be scheduled in 2004/2008, the study of these reactions can be extended to both higher luminosities but also to much higher invariant masses, hithero unexplored. We quote J. D. Bjorken [9]: It is an important portion (of the FELIX program at LHC (7]) to tag on Weizsaecker Williams photons (via the nonobservation of completely undissociated forward ions) in ion-ion running, creating a high luminosity $\gamma \gamma$ collider.

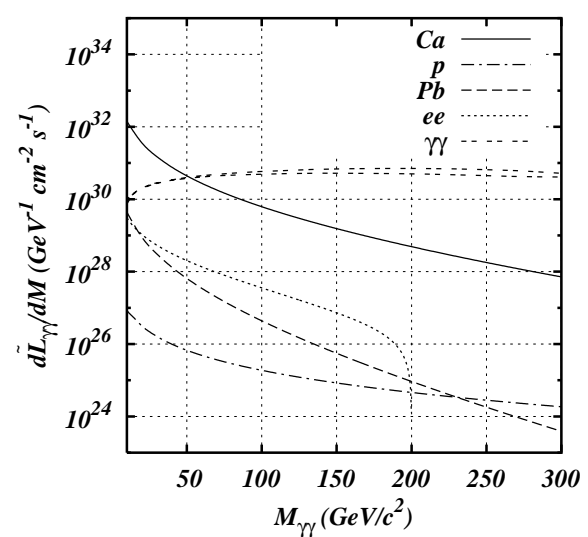

Figure 2. Comparison of the effective $\gamma \gamma$-Luminosities $\left(d \tilde{L}_{\gamma \gamma} / d M=L_{A A} \times\right.$ $\left.d L_{\gamma \gamma} / d M\right)$ for different ion species. For comparison the same quantity is shown for LEP2 ("ee") and a future NLC/PLC (next linear collider/photon linear collider, " $\gamma \gamma$ "), where photons are obtained by laser backscattering; the results for two different polarizations are shown.

In Fig. 2 we compare the effective $\gamma \gamma$ luminosities, that is, the product of the beam luminosity with the two-photon luminosity $\left(L_{A A} \times d L_{\gamma \gamma} / d M\right)$ for various collider scenarios. We use the following collider parameters: LEP2: $E_{e l}=$ $100 \mathrm{GeV}, L=10^{32} \mathrm{~cm}^{-2} \mathrm{~s}^{-1}$, NLC/PLC: $E_{e l}=500 \mathrm{GeV}, L=2 \times 10^{33} \mathrm{~cm}^{-2} \mathrm{~s}^{-1}, \mathrm{~Pb}-$ $\mathrm{Pb}$ heavy-ion mode at LHC: $\gamma=2950$, $L=10^{26} \mathrm{~cm}^{-2} \mathrm{~s}^{-1}$, Ca-Ca: $\gamma=3750$, $L=4 \times 10^{30} \mathrm{~cm}^{-2} \mathrm{~s}^{-1}$,p-p: $\gamma=7450$, $L=10^{34} \mathrm{~cm}^{-2} \mathrm{~s}^{-1}$. In the Ca-Ca heavy ion mode, higher effective luminosities can be achieved as, e.g., in the $\mathrm{Pb}-\mathrm{Pb}$ mode, since higher AA luminosities can be reached there. Since the event rates 
are proportional to the luminosities, and interesting events are rare (see also below), we think that it is important to aim at rather high luminosities in the ion-ion runs. This should be possible, especially for the medium heavy ions like Ca. For further details see 10,11.

\section{2. $\gamma A$ collisions}

The interaction of quasireal photons with protons has been studied extensively at the electron-proton collider HERA, where the quasi-real photons from the electron (positron) beam are used. Similar and more detailed studies will be possible at the relativistic heavy ion colliders RHIC and LHC using the larger flux of quasireal photons from one of the colliding nuclei. In the photon-nucleon subsystem, one can reach invariant masses $W_{\gamma N}$ up to $W_{\gamma N, \max }=\sqrt{4 W_{\max } E_{N}} \approx$ $0.8 \gamma A^{-1 / 6} \mathrm{GeV}$. For $\mathrm{Pb}$ at $\operatorname{LHC}(\gamma=$ 2950) one obtains $950 \mathrm{GeV}$ and even higher values for Ca. Thus one can study physics quite similar to the one at HERA, with nuclei instead of protons.

Photon-nucleon physics includes many aspects, like the energy dependence of total cross-sections, diffractive and nondiffractive processes. An important subject is the elastic vector meson production $\gamma p \rightarrow V p$ (with $V=\rho, \omega, \phi, J / \Psi, \ldots$ ). Here one gets insight into the interface between perturbative QCD and hadronic physics. Elastic processes, that is, the proton remaining in its ground state, as well as inelastic ones, are of interest. For the hard exclusive photoproduction of $J / \Psi$ mesons, recent data from HERA have shown a rapid increase of the total cross section with $W_{\gamma N}$, in agreement with perturbative QCD.

Such studies could be extended to photon-nucleus interactions at RHIC, thus complementing the HERA studies. Of special interest is the coupling of the photon of one nucleus to the Pomeronfield of the other nucleus. Such studies are envisaged for RHIC, see [12]. Estimates of vector meson production in photonnucleon processes at RHIC and LHC are given in [2]. In $A A$ collisions one can have incoherent photoproduction on the individual $A$ nucleons as well as coherent one. Shadowing effects will occur in the nuclear environment and it will be interesting to study these. For the coherent production one expects an $A^{4 / 3}$ dependence at large momentum transfer, in contrast to the $A^{2}$ dependence, one sees, e.g., in low energy $\nu \mathrm{A}$ scattering. Calculations 13 for RHIC show an $A$-dependence between these two extremes. In this context, RHIC and LHC can also be thought of as vector meson factories [13].

\section{Photon-Photon Physics}

Up to now photon-photon scattering has been mainly studied at $e^{+} e^{-}$colliders. The traditional range of invariant masses has been the region of mesons, ranging from $\pi^{0}\left(m_{\pi^{0}}=135 \mathrm{MeV}\right)$ up to about $\eta_{c}\left(m_{\eta_{c}}=2980 \mathrm{MeV}\right)$. Also the total $\gamma \gamma \rightarrow$ hadron cross-section was studied, e.g., at LEP2 14,15. In contrast to $e^{+} e^{-}$colliders, the photons from heavy ions have always $Q^{2} \lesssim 1 / R^{2}$ due to the coherence condition and are therefore in 
all these cases quasireal ("untagged").

Two-photon collisions give access to most of the $C=+1$ mesons. In principle $C=-1$ vector mesons can be produced by the fusion of three or more equivalent photons. But this effect is smaller than the contribution coming from $\gamma$-A collisions (see above), even for nuclei with large $Z$ (see [2]).

While the $\gamma \gamma$ invariant masses, which will be reached at RHIC, will mainly be useful to explore QCD at lower energies, the $\gamma \gamma$ invariant mass range at $\mathrm{LHC}-$ up to about $100 \mathrm{GeV}$ - will open up new possibilities. This includes the discovery of the Higgs-boson in the $\gamma \gamma$-production channel or new physics beyond the standard model, like supersymmetry or compositeness.

A number of calculations have been made for a medium heavy standard model Higgs [16-19]. Chances of finding the standard model Higgs in this case are only marginal [8]. An alternative scenario with a light Higgs boson was, e.g., given in 20] in the framework of the "general two Higgs doublet model", which allows for a very light particle in the few $\mathrm{GeV}$ region. The authors of [20] proposed to look for such a light neutral Higgs boson at the proposed low energy $\gamma \gamma$-collider. We want to point out that the LHC Ca-Ca heavy ion mode would also be very suitable for such a search.

One can also speculate about new particles with strong coupling to the $\gamma \gamma$ channel. Large $\Gamma_{\gamma \gamma}$-widths will directly lead to large $\gamma \gamma$ production cross-sections, see [21,22]. Since the $\gamma \gamma$-width of a reso- nance is mainly proportional to the wave function at the origin, huge values can be obtained for very tightly bound systems. Composite scalar bosons at $W_{\gamma \gamma} \approx$ $50 \mathrm{GeV}$ are expected to have $\gamma \gamma$-widths of several MeV [21,22]. The search for such kind of resonances in the $\gamma \gamma$-production channel will be possible at LHC.

In Refs. [23,24] $\gamma \gamma$-processes at $p p$ colliders were studied. It was observed that non-strongly interacting particles (sleptons, charginos, neutralinos, and charged Higgs bosons) are difficult to detect in hadronic collisions as Drell-Yan and $g g$ fusion yield only low production rates for such particles. Therefore producing such particles in $\gamma \gamma$ interactions was examined. Clean events can be expected, if the protons do not break up in the photon emission process. In 23] it was also pointed out, that at the high luminosity for $p p$ collisions at LHC about 16 minimum bias events per bunch crossing are expected. These hadronic background events are not a big concern in heavy ion collisions, due to their much smaller luminosities.

Similar considerations for new physics are also made in connection with the planned $e A$ collider at DESY (Hamburg). Again, the coherent field of a nucleus gives rise to a $Z^{2}$ factor in the cross-section for photon-photon processes in $e A$ collisions [25].

An overview of the expected events rates for $\mathrm{Ca}-\mathrm{Ca}$ collisions at $\mathrm{LHC}$ is given in Figure 3. Both production of resonances and continuum states are shown.

An interesting topic in itself is the $e^{+} e^{-}$ pair production. The fields are strong 

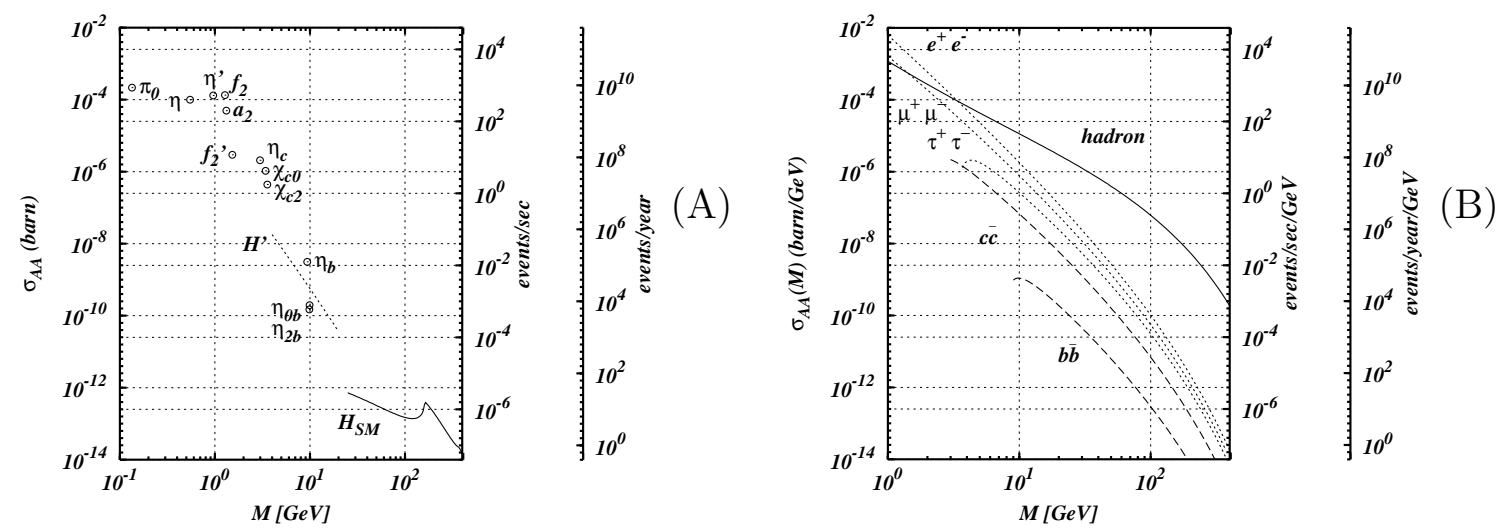

Figure 3. Overview of the total cross section and production rates of resonances (A) and continuum states $(\mathrm{B})$ in Ca-Ca collisions at the LHC using a beam luminosity of $L_{A A}=4 \times 10^{30} \mathrm{~cm}^{-2} \mathrm{~s}^{-1}$ and $10^{7} \mathrm{~s}$ per year. $H_{S M}$ denotes the standard model Higgs, $H^{\prime}$ the one in the "general two Higgs doublet model". For further details see [2].

enough to produce multiple pairs in a single collisions. A discussion of this subject together with calculations within the semiclassical approximation can be found in [26,27, see also [28].

\section{Conclusion}

Electromagnetic processes, that is, photon-photon and photon-hadron collisions, are an interesting option for heavy ion colliders, complementing the program for central collisions. It is the study of "silent events", with relatively small multiplicities and a small background. The method of equivalent photons is a well established tool to describe these kinds of reactions; effects arising from the more complex structure of the ions are well under control. Remaining uncertainties coming, e.g., from triggering can be eliminated by using a luminosity monitor from muonor electron-pairs [5]. A trigger for periph- eral collisions is essential in order to select photon-photon events. Such a trigger seems to be possible based on the survival of the nuclei after the collision and the use of the small transverse momenta of the produced system 12]. Difficult to judge quantitatively at the moment is the influence of strong interactions in grazing collisions, i.e., effects arising from the nuclear stratosphere and Pomeron interactions.

The high photon fluxes open up possibilities for studies up to energies hitherto unexplored. At RHIC the invariant mass range extends up to several $\mathrm{GeV}$. At the LHC one also has the possibility to study new physics in the $100 \mathrm{GeV}$ range.

Peripheral collisions using PhotonPomeron and Pomeron-Pomeron collisions, that is, diffractive processes are an additional application. They use essentially the same triggering conditions and therefore one should be able to record them at the same time as photon-photon 
events.

\section{REFERENCES}

1. F. Vannucci, in $\gamma \gamma$ Collisions, Proceedings, Amiens 1980, Vol. 134 of Lecture Notes in Physics, ed. by G. Cochard (Springer, 1980).

2. G. Baur, K. Hencken, and D. Trautmann, Topical Review, J. Phys. G 24, 1657 (1998).

3. F. Krauss, M. Greiner, and G. Soff, Prog. Part. Nucl. Phys. 39, 503 (1997).

4. J. Nystrand, in Proc. of the Lund workshop on photon interactions and the photon structure, Lund, September 10-13, 1998, ed. by G. Jarlskog and T. Sjostrand (Lund, 1998).

5. Serguei Sadovsky, CERN-Note ALICE/92-07, 1992.

6. K. Hencken, Yu. V. Kharlov, G. V. Khaustov, S. A. Sadovsky, and V. D. Samoylenko, TPHIC, event generator of two photon interactions in heavy ion collisions, IHEP-96-38, 1996.

7. K. Eggert et al., FELIX Letter of Intent, CERN/LHCC 97-45, LHCC/I10, 1997.

8. G. Baur, K. Hencken, D. Trautmann, S. Sadovsky, and Yu. Kharlov, Photon-Photon Physics with heavy ions at CMS, CMS Note 1998/009, 1998.

9. J. D. Bjorken, Nucl. Phys. B (Proc. Suppl.) 71, 484 (1999).

10. D. Brandt, K. Eggert, and A. Morsch, CERN AT/94-05(DI), 1994.

11. K. Hencken, D. Trautmann, and G. Baur, Z. Phys. C 68, 473 (1995).
12. S. Klein and E. Scannapieco, in 6th Conf. on the Intersections of Particle and Nuclear Physics, May 1997, Big Sky, Montana, STAR Note 298, LBNL-40495, 1997.

13. S. Klein and J. Nystrand, to appear in Phys. Rev. C,hep-ph/9902259, 1999.

14. L3 collaboration, Phys. Lett. B 408, 450 (1997).

15. see contributions in session $\mathrm{C}$, these proceedings.

16. M. Drees, H. Ellis, and D. Zeppenfeld, Phys. Lett. B 223, 454 (1989).

17. B. Müller and A. J. Schramm, Phys. Rev. D 42, 3699 (1990).

18. E. Papageorgiu, Phys. Lett. B 352, 394 (1995).

19. J. Norbury, Phys. Rev. D 42, 3696 (1990).

20. D. Choudhury and M. Krawczyk, Phys. Rev. D 55, 2774 (1997).

21. F. M. Renard, Phys. Lett. B 126, 59 (1983).

22. U. Baur, H. Fritzsch, and H. Faissner, Phys. Lett. B 135, 313 (1984).

23. M. Drees, R. M. Godbole, N. Nowakowski, and S. D. Rindami, Phys. Rev. D 50, 2335 (1994).

24. J. Ohnemus, T. F. Walsh, and P. M. Zerwas, Phys. Lett. B 328, 369 (1994).

25. M. Krawczyk and B. B. Levtchenko, in Proc. Future Physics at HERA 19951996, Ed. G. Ingelmann, A. de Roeck, R. Klanner.

26. K. Hencken, D. Trautmann, and G. Baur, Phys. Rev. A 51, 1874 (1995).

27. A. Alscher, et al., Phys. Rev. A 55, 396 (1997).

28. V. Serbo, these proceedings. 